\title{
Paraneoplastic myositis: case report and review of literature
}

\author{
Authors: Dana Ershaid, Helen Wilson and Lindsay George
}

\section{Aims}

To present a rare association of squamous cell carcinoma of the tonsil with myositis with a review of similar cases reported in the literature.

\section{Methods}

We present a 69 -year-old male patient admitted to hospital with an 8-week history of lethargy, proximal lower limb weakness and weight loss. On systemic review there was nothing of note.

\section{Results}

He was found to have elevated creatine kinase $(12,208)$; in view of his presenting complaint, he was investigated for a working diagnosis of polymyositis. MRI of thighs showed characteristic inflammation of the affected muscle group. Muscle biopsy was obtained and he was commenced on high-dose steroids without much improvement clinically or biochemically. Muscle biopsy results showed evidence of necrotising myopathy. This was further supported with electromyography studies, which were again suggestive of the diagnosis. While on the ward he had daily lung function tests to assess for respiratory muscle weakness. He went on to develop upper limb and phayrngeal weakness, for which he underwent a barium swallow and CT neck that revealed a right sided tonsillar mass for which he had laser excision and neck dissection. Histopathology came back to show right tonsil squamous cell carcinoma with neck metastasis. During his hospital stay, he complained of pleuritic chest pain with haemoptysis; CTPA was carried out and showed multiple bilateral pulmonary emboli and right lower lobe changes in keeping with acute infarction. He was started on therapeutic-dose enoxaparin. Upon resection of the tumour, his creatine kinase levels dropped significantly from $>10,000$ to $<300$ and his mobility continued to improve gradually, but unfortunately due to the necrotising myopathy his swallowing has failed to improve and he remains PEG fed.

\section{Conclusions}

Inflammatory myopathies are rare autoimmune conditions which carry paraneoplastic associations. Patients with polymyositis or dermatomyositis who present with unusual features or risk factors such as slow treatment response, aged older than 45 , male sex and rapidly progressive symptoms should be screened for malignancy. 